\title{
ARTÍCULOS
}

\section{Nuevas aplicaciones dentales de vidrio bioactivo nanoestructurado y sus composites*}

\author{
David Don López, ** Alessandro Polini, ${ }^{* *}$ \\ Antoni P. Tomsia**
}

RESUMEN: Para la mejora de los tratamientos de traumas dentales u óseos y diferentes enfermedades, como pueden ser la osteoporosis, el cáncer o distintas infecciones, los científicos se esfuerzan en colaborar conjuntamente con médicos para poder diseñar y probar nuevos biomateriales para la regeneración del tejido perdido o dañado. Desarrollado hace más de 50 años, el vidrio bioactivo (BG, por sus siglas en inglés) empieza a ser recientemente uno de los biomateriales más prometedores del panorama científico, como consecuencia del descubrimiento de inusuales propiedades que provocan respuestas biológicas específicas en el cuerpo. Entre estas importantes propiedades está la capacidad del BG de formar fuertes interacciones, tanto con tejidos blandos como con los duros, así como el proceso de liberación de iones en la disolución. Desarrollos recientes en nanotecnología han introducido nuevas oportunidades para la ciencia de materiales, aplicados a terapias dentales y óseas de diferentes tipos. Por ejemplo, las aplicaciones para el BG se expanden a medida que es posible controlar con mayor precisión las estructuras producidas con él y las propiedades fisicoquímicas del propio material a nivel molecular. En la presente publicación se hace un estudio de cómo las propiedades de estos materiales han sido mejoradas con la llegada de la nanotecnología, y cómo estos avances están produciendo resultados realmente prometedores en la regeneración de tejido duro y el desarrollo del innovador sistema de liberación de medicamentos basado en el BG.

PaLABAS Clave: vidrio bioactivo, biomateriales, regeneración de tejido duro.

AвSTRACT: In order to achieve the improvement of dental and bone treatments for different kind of trauma or diseases, e.g., osteoporosis, cancer or infections, material scientists are joining their efforts and working together with clinicians towards the development of new biomaterials. Developed more than 50 years ago, bioactive glass (BG) has recently gained attention and is considered promising biomaterial in the scientific scene, thanks to the discovery of its unusual properties that promote biological responses inside of the human body. Among their important properties, the BG is able to form strong interactions with both soft and hard tissue, also due to the release of ions upon dissolution. In material science, the last developments in nanotechnology have showed new opportunities and directions in several application fields, including for dental and bone therapies. For example, new applications for BG are available as more control on the structures and physicochemical properties of materials at the molecular level is possible. Here, we summarize how these material properties have been improved by the use of nanotechnology, and how these developments are leading to really promising results in the field of hard-tissue regeneration and in the progress of innovative drug delivery systems based on BG.

KEY WORDS: bioactive glass, biomaterials, hard-tissue regeneration.

\footnotetext{
* Este trabajo ha sido financiado por los National Institutes for Health and Dental and Craniofacial Research (NIH/NIDCR), con la beca número 1R01DE015633.

** Materials Sciences Division, Lawrence Berkeley National Laboratory, Berkeley, CA 94720.

Correo-e de Alessandro Polini: (apolini@lbl.gov).
} 


\section{INTRODUCCIÓN}

En los tratamientos de traumas o de enfermedades dentales u óseas, como puede ser la osteoporosis, el cáncer o diferentes tipos de infecciones, los materiales sintéticos biocompatibles son habitualmente utilizados para remplazar los tejidos dañados. Sin embargo, este tipo de materiales no suelen tener propiedades químicas, biológicas o físicas lo suficientemente similares al tejido original, provocando problemas y, en ocasiones, la necesidad de retratamiento para poder arreglar la situación. Para llegar al objetivo de la completa recuperación del paciente es esencial desarrollar nuevos materiales activos que sean capaces de interactuar con los tejidos circundantes, mejorando y dirigiendo la completa recuperación del tejido.

Para conseguir este objetivo, diferentes grupos científicos de investigación en el área de la ciencia de materiales están focalizando sus esfuerzos en el vidrio bioactivo (BG, por sus siglas en inglés) y otro tipo de composites con BG. Los BGs son materiales con capacidad de inducir respuestas biológicas específicas después de su implantación [1]. Concretamente, esta bioactividad es conseguida gracias a la formación en su superficie de capas de hidroxiapatita (HA), capaz de establecer fuertes enlaces tanto con el tejido blando como con el duro, y libera iones después de disolverse [2]. Además, los avances técnicos en el procesamiento del BG han desembocado en el desarrollo de vidrios bioactivos mesoporosos (MBG), materiales que extienden el potencial del BG hacia el diseño de sistemas innovadores para la liberación de medicamentos [3].

Estudios recientes han demostrado también que las herramientas de la nanotecnología pueden ser utilizadas para mejorar el funcionamiento de los materiales actualmente utilizados para tratar problemas con el crecimiento óseo o para solucionar diferentes problemas dentales [4-6]. Estas nuevas técnicas, las cuales tienen en cuenta la compleja y jerárquica estructura de los dientes y los huesos, pueden imitar la nanoestructura original de los tejidos humanos y así desarrollar nuevos implantes que podrían ser cuidadosamente diseñados a diferentes escalas [7]. Mientras tanto, la aplicación de la nanotecnología para tratamiento, diagnóstico, monitorización y control de los sistemas biológicos (recientemente nombrada como "nanomedicina" por el Instituto Nacional de Salud estadounidense, el NIH) ofrece una oportunidad única para un verdadero diseño racional y una liberación de medicamentos focalizada, minimizando los efectos adversos para los tejidos adyacentes [8].

Aquí se muestra cómo la nanotecnología puede ser aplicada para modificar y mejorar los materiales de BG. Después de un pequeño resumen de las principales propiedades del BG y de un estudio de la relación entre la composición y la bioactividad, se describirán los últimos avances y estudios en el uso de materiales nanoestucturados con presencia de BG para la regeneración de tejido duro y el desarrollo de sistemas con liberación de medicamentos.

\section{VIDRIO BIOACTIVO}

El BG puede ser formado usando dos métodos diferentes. Por un lado, el tradicional fundido-enfriado, utilizado para aplicaciones comerciales del BG, en donde se emplean temperaturas superiores a $1,300{ }^{\circ} \mathrm{C}$ para fundir todos los óxidos en un crisol de platino, mientras que barras o monolitos de grafito o agua son utilizados para el en- 
friamiento. Por otro lado, el método más moderno desarrollado se basa en el sol-gel, una síntesis química en la que se emplean precursores de sílice para formar y aglomerar nanopartículas (NPs) dentro de un gel a temperatura ambiente [9]. El vidrio es formado después de secar y calentar este gel, que es una red húmeda y orgánica de enlaces covalentes de sílice. Una diferencia notable entre los vidrios obtenidos a partir de estos métodos es la presencia de nanoporosidad empleando la técnica sol-gel, lo cual conlleva a un aumento de la superficie específica y mejora la respuesta celular al material [10]. Las composiciones obtenidas a través del método sol-gel muestran menos componentes porque algunos óxidos, como el $\mathrm{Na}_{2} \mathrm{O}$, muy comúnmente utilizado en el proceso de fundido-enfriado para mejorar el proceso disminuyendo la temperatura del punto de fusión de la mezcla, no son necesarios para este método. Más detalles pueden ser encontrados en los últimos estudios [2,11,12].

Los MBGs altamente ordenados son comúnmente sintetizados a través del método sol-gel por medio de bloques de polímeros no iónicos, que son utilizados para dirigir la formación de la estructura durante el proceso de auto ensamblaje que se produce al inducir la evaporación del agua del gel [3]. A pesar de que los BGs convencionales muestran una estructura mesoporosa debido a la distribución aleatoria de $\mathrm{CaO}$ dentro de la red de $\mathrm{SiO}_{2}$, el auto ensamblaje de los surfactantes en el MBGs conlleva la uniformización de los mesoporos, con tamaños entre 2 y $50 \mathrm{~nm}$ así como estructuras ordenadas. Además del control sobre la composición del MBG, este proceso de auto ensamblaje ofrece la oportunidad de establecer el tamaño de poro y la estructura final [3]. Cuando es comparado con la arquitectura del BG no mesoporoso, el incremento de la superficie específica en la estructura del MBG otorga mejores cualidades, tanto in vivo como in vitro, como la bioactividad, la degradación y las propiedades de liberación de fármacos [13,14].

\section{ESTRUCTURA ATÓMICA DEL BG}

La bioactividad de los materiales basados en el BG, que se mineralizan y forman capas de hidroxicarbonato de apatito (HCA), depende del control preciso de la composición y de la estructura atómica del vidrio, así como de las propiedades osteogénicas, angiogénicas y bactericidas de sus disoluciones. La estructura atómica de los vidrios de sílice está determinada por las propiedades electrónicas de los átomos de silicio que, al ser combinados con átomos de oxígeno, forman tetraedros conectados por medio de enlaces de oxígeno de la manera Si-O-Si [15]. Los cationes que modifican esta red (como el sodio o el calcio) pueden interrumpir la estructura estableciendo otro tipo de enlaces. En general, los BGs con un alto contenido en sílice tienen redes mejor interconectadas, lo cual conlleva a una disolución y una bioactividad más lenta. Además del uso de cationes para modificar la estructura atómica, la adición de fósforo puede inducir la repolimerización de la red de silicatos, produciendo efectos similares a la extracción de cationes, contrarrestando las cargas e induciendo una distribución heterogénea de ellos [16].

Un parámetro importante para la predicción de la bioactividad de un vidrio es la conectividad de la red, $\mathrm{N}_{c}$, ésta indica el número de enlaces por átomo de silicio. Vidrios con valores de $\mathrm{N}_{\mathrm{c}}$ mayores que 2.6 no son propensos a ser bioactivos, pues poseen una gran resistencia a disolverse [17]. En los vidrios sol-gel el valor de $\mathrm{N}_{c}$ es, generalmente, menor que el calculado a partir de la composición nominal debido a la 
presencia de grupos hidroxilos en su composición [2]. A pesar de que el proceso de secado elimina la mayoría de los grupos $-\mathrm{OH}$ provocando una mayor formación de enlaces $\mathrm{O}-\mathrm{Si}-\mathrm{O}$, algunos permanecen atrapados en la red, reduciendo su conectividad.

\section{BIOACTIVIDAD DEL BG: FORMACIÓN DE HCA}

La capacidad del BG de adherirse al hueso es atribuida a diferentes procesos, como la formación de una capa de HCA capaz de interactuar fuertemente con las fibras de colágeno o con el propio hueso dañado, la absorción de proteínas, la incorporación de moléculas de colágeno y la inducción de diferenciación y adhesión de los osteoblastos [18]. La capa de HCA es formada como resultado de varias reacciones en la superficie del BG de la siguiente manera [19]: (1) incremento del pH debido a un rápido intercambio iónico entre los cationes de la propia estructura con $\mathrm{H}^{+}$de la solución, lo cual conlleva a una hidrólisis de los grupos de sílice y la creación de grupos de silanol (Si-OH); (2) el incremento de $\mathrm{pH}$ provoca el ataque de los iones $\mathrm{OH}^{-}$a la estructura vítrea de $\mathrm{SiO}_{2}$, rompiendo los enlaces $\mathrm{Si}-\mathrm{O}-\mathrm{Si}$; (3) la condensación y polimerización de una capa de 1-2 $\mu \mathrm{m}$ de $\mathrm{SiO}_{2}$ amorfo exento de $\mathrm{Na}^{+} \mathrm{y} \mathrm{Ca}^{2+}$; (4) fomento de la disolución del vidrio y migración del $\mathrm{Ca}^{2+}$ y del $\left(\mathrm{PO}_{4}\right)^{3-}$ hacia la capa de $\mathrm{SiO}_{2}$ amorfo, creando una capa de fosfato cálcico amorfo (ACP); y (5) a medida que el vidrio se va disolviendo, la capa de ACP integra los iones $(\mathrm{OH})^{-}$y $\left(\mathrm{CO}_{3}\right)^{2-}$ desde la disolución y cristaliza como una capa de HCA.

\section{BIOACTIVIDAD DEL BG: PRODUCTOS DE LA DISOLUCIÓN IÓNICA}

Los primeros estudios sobre las propiedades biológicas de los productos de la disolución iónica procedentes del BG se han centrado en el Si, Ca y P, y muestran cómo estos materiales pueden modificar la expresión génica de diferentes genes en células del tipo osteoblastos [20]. De esta manera, otros efectos has sido investigados, tanto in vitro como in vivo, haciendo especial hincapié en factores como la angiogénesis, la actividad bactericida y el proceso inflamatorio. Más adelante, los últimos estudios en este campo serán revisados para describir tales efectos (para ejemplos adicionales consultar [21]).

Estructuras macroporosas de MBG dopadas con Mg, Zn o Sr son producidas por medio de moldes de espuma de polímeros [22]. Como se ha demostrado con los estudios in vivo usando células mesenquimales de médula de hueso de rata (rBMSCs), esta adición introduce efectos no citotóxicos, con una liberación sostenida de $\mathrm{Ca}, \mathrm{P}, \mathrm{Si}, \mathrm{Mg}$, Zn y Sr desde las piezas hacia el medio de cultivo, favoreciendo la proliferación celular y la actividad de la fosfatasa alcalina (ALP). En otro estudio, la liberación de B por medio de piezas de MBG funcionalizados con dexametasona bórica (DEX) conllevan un incremento de la actividad de la ALP y de la expresión de genes osteogénicos sinérgicamente con la liberación de DEX [23]. Las estructuras de MBG dopadas con Fe magnético, muy útiles como plataforma hipertérmica en el tratamiento de tumores óseos malignos, han sido desarrollados e investigados in vitro, mostrando una mejora en la actividad mitocondrial, así como en las expresiones génicas óseas (ALP y osteocalcina) en BMSCs humanos (hBMSCs) [24]. El incremento de la adhesión y de la viabilidad de los hBMSCs también es conseguido por medio de estructuras dopadas con $\mathrm{Zr}$, 
las cuales muestran una mayor resistencia a la compresión y menor solubilidad al ser comparadas con estructuras no dopadas, mientras que la capacidad de formar apatito se mantiene [25]. Un interesante y reciente estudio reporta que la introducción de Co en las piezas de MBG produce la inducción a un medio hipóxico para mejorar la angiogénesis a través de la secreción de mayores cantidades del factor de crecimiento del endotelio vascular (VEGF), el factor $1 \alpha$ que induce a la hipoxia y expresiones génicas óseas de hBMSCs [26]. Resultados similares pueden ser logrados con la incorporación de $\mathrm{Cu}$ en piezas de MBG [27]. La introducción de Li en los implantes de MBG tiene un efecto específico en la formación del cemento dental, el tejido mineral producido por los cementoblastos durante la formación de la raíz dental. El cemento dental es el responsable de la unión del ligamento periodontal a las raíces y rodear el hueso alveolar influenciando la proliferación y la diferenciación cementogénica de las células derivadas del ligamento periodontal humano [28].

Además de los estudios de los efectos específicos del BG y el MBG en la regeneración ósea y la angiogénesis, varios artículos se han centrado en las propiedades bactericidas de los materiales BG, resultantes de su disolución y la liberación de iones al medio, para el tratamiento o prevención de infecciones periodontales [21,29]. Por ejemplo, partículas de $\mathrm{BG}$ dopadas con $\mathrm{Ag}_{2} \mathrm{O}$ pueden ser sintetizadas por medio del método sol-gel y usadas no sólo como bacteriostático, sino también como material bactericida [30]. Estas propiedades antibacterianas son probablemente debidas a la lixiviación de los iones de plata desde la matriz vítrea y su acumulación por las bacterias, donde interfieren con diferentes mecanismos celulares que todavía no han sido completamente entendidos [30]. El-Kady y demás produjeron NPs de BG dopadas con $\mathrm{Ag}_{2} \mathrm{O}$ mostrando una liberación continuada de iones de plata durante dos semanas, capaces de inhibir el crecimiento de Staphylococcus aureus y Escherichia coli.

\section{REGENERACIÓN DE TEJIDOS DUROS}

Los huesos y los dientes son órganos extremadamente complejos con una combinación de diferentes tejidos duros (hueso trabecular y compacto, esmalte dental, dentina o el cemento dental) y tejidos blandos (médula ósea, pulpa dental o el ligamento periodental) en una estructura jerárquica única, donde la nano y la mesoescala son igual de importantes, con una gran combinación de fenómenos complejos como las interacciones biomoleculares, el intercambio de nutrientes o el transporte de fluidos. Autoinjertos, alotransplantes y xenotransplantes han sido utilizados como sustitutos óseos cuando el proceso de recuperación o remodelado biológico no era capaz de curar los defectos óseos causados por diferentes traumas, extracciones tumorales y distintas enfermedades congénitas. Para poder evitar las limitaciones que cada uno de estos tejidos de los diferentes donantes tienen, biomateriales como metales, polímeros y composites están siendo utilizados desde los años 50 [32]. Biomateriales de tercera generación, disponibles en forma de polvo, soluciones o partículas están actualmente siendo investigados con el objetivo de promover la rápida regeneración del tejido en el paciente [18].

Como ha sido descrito en la sección anterior, el BG es muy efectivo al provocar repuestas celulares específicas. Como resultado, estos materiales son ampliamente usados en ingeniería de tejidos duros, tanto como material aislado como fase inorgánica en composites híbridos [11, 12, 33, 34]. Recientemente, materiales nanoestruc- 
turados con base de BG han sido creados con diferentes métodos de procesamiento (congelamiento unidireccional de suspensiones, electrospinning, procesado sol-gel, replicación de espuma polimérica, técnicas de microemulsión, o enlace térmico de partículas o fibras) en la forma de piezas tridimensionales (3D) como NPs o nanorrecubrimientos, demostrando propiedades mecánicas similares y comparables a las del hueso natural $[11,33]$. El aumento de la superficie específica de los productos del BG nanoescalado tiene dos efectos importantes: una disolución más rápida y la consecuente liberación de iones; y una mayor adsorción proteica. Estos efectos tienen el potencial de desembocar en una mejora de la bioactividad así como en nuevas e inesperadas aplicaciones biomédicas.

\section{BG COMO MATERIAL EN SOLITARIO}

Para el tratamiento de defectos óseos de gran tamaño, es habitual el uso de moldes 3D como marco para guiar las células óseas en el proceso regenerativo con el objetivo de estimular los mecanismos de curado fisiológico dentro del cuerpo humano [35]. Este tipo de piezas suelen ser llenadas con MSCs antes de su implantación, y muestran una porosidad adecuada para permitir la formación de nuevos vasos sanguíneos, que son fundamentales para la supervivencia del hueso a regenerar. Estructuras tridimensionales con sobresalientes propiedades mecánicas (136 MPa como resistencia a la compresión, comparable a la del hueso cortical) y una alta porosidad (60\%, también similar a la que presenta el hueso cortical) son fabricadas con vidrio 6P53B por medio de ensamblado directo de tinta, conservando una excelente bioactividad in vitro [39]. Después de la inmersión en fluido corporal simulado (SBF) durante 3 semanas, toda la pieza es recubierta por cristales de HA a nanoescala. La resistencia a la compresión desciende hasta valores de 77 MPa debido a la degradación del material, pero sus propiedades mecánicas siguen estando muy por encima del hueso trabecular, con lo que su aplicación sigue siendo plausible para el tratamiento de defectos óseos de baja carga mecánica [39,40]. La impresión 3D puede ser utilizada con suspensiones de MBG para la formación de estructuras porosas distribuidas jerárquicamente y con buena resistencia mecánica, que además preservan excelentemente la habilidad de mineralización del apatito y las propiedades de liberación sostenida de fármacos [42,50]. El congelamiento unidireccional de una suspensión de vidrio 13-93 con base de canfeno es empleada para fabricar estructuras anisotrópicas porosas en 3D con muy buenas propiedades mecánicas (47 MPa) y una alta porosidad (50\%) [36]. Utilizando la técnica del moldeado de espuma de gel se producen estructuras de vidrio silicato ICIE 16, realizadas sin la cristalización del vidrio (con lo que no pierden bioactividad). El gran tamaño de poro que presentan (con una media de $379 \mu \mathrm{m}$ ) favorece la formación de nuevos vasos sanguíneos, mientras que su resistencia mecánica a la compresión se reduce al mínimo del hueso trabecular (1.9 MPa) [41]. La misma técnica permite la fabricación de estructuras de silicato BG con porosidades similares (con una media de $372 \mu \mathrm{m}$ ) y con la capacidad de soportar la diferenciación de los tres diferentes tipos de células que están principalmente relacionadas con el buen desarrollo del sistema vascular óseo: macrófagos de ratón (línea celular C7), que se diferencian en osteoclastos multinucleados TRAP've; preosteoblastos de ratón (MC3T3-E1), que depositan nódulos mineralizados en la superficie; y células endotélicas aórticas bovinas, que crean estructuras tubulares [51]. Recubrimientos de BG de nanotamaño están siendo es- 
tudiadas por su potencial para mejorar la estabilidad de los implantes metálicos por medio del enlazamiento de éstos con el propio hueso, así se evitaría la formación de quistes fibrosos después de su implementación [52], aunque la rápida disolución del BG puede llevar a largo plazo a la inestabilidad del implante metálico.

Recientemente, la técnica de microspinning ha sido utilizada en un esfuerzo por imitar la arquitectura de la matriz extracelular (ECM) compuesta por haces de fibras de proteínas y glicanos de tamaños entre 100 y $500 \mathrm{~nm}$. Con esta técnica, es posible producir estructuras de nanofibras de una manera rápida y controlable, permitiendo y dirigiendo a las células óseas a que proliferen y se diferencien en múltiples tipos [7, $53,54]$. Fibras de silicato BG de tamaño submicrométrico han sido producidas y sus propiedades mecánicas fueron estudiadas por medio de un nanoindentador y los resultados obtenidos muestran un relativamente alto módulo elástico (5.5 GPa), muy cerca del valor del hueso trabecular [55]. La zona hueca entre las fibras de MBG fue producida por medio del uso de una fase polimérica como núcleo del material, de fácil eliminación por incinerado durante la estabilización para dejar una entramado de puro BG [56, 57]. Nanofibras de BG 45S5 fueron también producidas por medio de tecnología láser de spinning, evitando el posterior tratamiento térmico o aditivos químicos para preservar su bioactividad en SBF.

Además del uso de estructuras tridimensionales, la posibilidad de utilizar gránulos o partículas para rellenar y reparar pequeños defectos de una manera rápida y controlable ha sido profundamente investigada por cirujanos ortopédicos y dentistas desde hace tiempo. Lanzado en 1993, para la reparación de defectos en la mandíbula en enfermedades periodontales, el Perioglass $®$ (actualmente vendido por NovaBone Products LCC, Alachua, FL) fue el primer producto de BG 45S5 microparticulado que evitaba la resorción del hueso alveolar en la mandíbula después de haber sustraído un diente o para esterilizar el canal de la raíz antes de fijar nuevos implantes dentales [29]. En los últimos años esfuerzos significativos han sido dirigidos a la investigación de NP de BG producidas principalmente por medio de síntesis con llama o por medio del proceso sol-gel. El objetivo es aprovechar su extremadamente gran ratio de superficie específica por volumen, consecuencia de su estructura nanoescalada [2]. Las NPs del BG 45S5, con un tamaño medio de partícula entre 20 y $60 \mathrm{~nm}$, han demostrado una gran actividad osteoblástica y una rápida transformación (después de un día) de BG a HCA nanocristalina [59]. NPs de silicato BG con diferentes tamaños medios de partículas (40-2000 $\mathrm{nm}$ ) preservan una liberación de silicio estable y permiten la unión y proliferación de las MSCs, con NPs de tamaños entre 40-800 y 40-180 nm mostrando las mayores formaciones de apatito y las mayores velocidades de proliferación, respectivamente. NPs de BG y sus productos iónicos también han sido propuestos para la regeneración del cemento dental [60]. Es interesante la posibilidad de utilizar estas NPs de BG como nanobloques para la construcción de macroestructuras ensambladas jerárquicamente, manteniendo intactas sus propiedades bioactivas y su gran nivel de porosidad.

Disponible comercialmente desde 2004, el BG 45S5 de NovaMin® (actualmente vendido por GlaxoSmithKline, UK) fue el primer producto de BG usado en pasta de dientes para tratar la hipersensibilidad en la dentina. A diferencia de otras pastas de dientes que incorporan químicos que provocan la anestesia temporal del nervio dental para evitar el dolor, estas NPs se pueden adherir a la dentina. Estudios clínicos [62] demuestran que fomentan la formación de una capa de HCA que ocluye los conductos tubulares de la dentina $(\approx 1 \mu \mathrm{m}$ de diámetro), permitiendo la remineralización de 
la dentina y aliviando el dolor por periodos de tiempo mayores. Persiguiendo el mismo objetivo, algunos investigadores han conseguido producir silicato MBG con tamaños de partículas de $40 \mathrm{~nm}$ para el tratamiento de la hipersensibilidad en la dentina, obteniendo resultados con una significativa reducción de la permeabilidad en la dentina [63]. Además, como ya se ha discutido en la última parte del artículo, pequeños medicamentos y factores de crecimiento pueden ser introducidos dentro de las partículas de BG y liberadas en las células después de que éstas hayan establecido contacto con las NPs.

\section{MATERIALES DE TIPO NANOCOMPOSITE DE BG Y POLÍMEROS}

Los nanocomposites formados por BG y polímeros son unos materiales bioactivos relativamente nuevos, que combinan la bioactividad y las buenas propiedades mecánicas del BG con la gran flexibilidad y capacidad de deformación bajo cargas de los polímeros. Estas características están disponibles, principalmente, para aplicaciones como estructuras 3D o para rellenado del propio hueso [33].

Utilizando colágeno del tipo I (COL) como adhesivo imitando el componente ECM y la fosfatidilserina (PS) como material con una gran afinidad por los iones de calcio, estructuras altamente porosas de BG-COL-PS fueron fabricadas por medio de la técnica de congelado-secado y probadas in vitro e in vivo [45]. En estudios de cultivos de MSC de ratas, estas piezas trifásicas demostraron adhesión celular, crecimiento y diferenciación osteogénica mayores, comparadas con el sistema bifásico BG-COL y, a su vez, el mayor grado de curación cuando eran introducidas MSCs en defecto de fémur de rata. Además, una combinación de COL y BG en forma de NPs fue estudiada, implantada como una pieza de hidrogel mineralizable y con semillas celulares, demostrando que conservaba la estructura nanofibrilar del COL así como la bioactividad del BG [64]. Una composición de BG obtenida por medio de sol-gel puede ser sometida a electrospinning y combinarse con COL para producir una matriz de composite con nanofibras, obteniendo así una magnífica bioactividad in vitro y una alta ALP después del cultivo de células osteoblásticas [37]. Las NPs de MBG pueden ser integradas en una disolución polimérica (policaprolactona, PCL) y seguidamente ser sometidas a un proceso de electrospinning, consiguiéndose así una estructura nanofibrilar más bioactiva que la matriz de PCL, en términos de adhesión, crecimiento y diferenciación celular in vitro [65]. Piezas tridimensionales de poli(D,L-lactida) rellenadas con partículas de micro y nanoescala, con una gran porosidad (81-93\%) y buenos rangos de resistencia mecánica (0.4-1.6 MPa), dentro de los parámetros del hueso trabecular, han sido probadas exitosamente in vivo [46], éstas son piezas bien implantadas con nuevo tejido formado, y muestran una vascularización mayor que las piezas de PDLLA, demostrando sus buenas propiedades osteogénicas y angiogénicas del BG.

Películas y recubrimientos de nanocomposites han sido desarrollados para mejorar las propiedades de materiales bioinertes como los metales, polímeros sintéticos o compuestos cerámicos. NPs de BG 45S5 (29 nm) fueron investigadas para preparar películas de composites poli(3-hidroxibutirato) por medio de la formación de una capa uniforme de HCA, después de la inmersión en SBF, mejorando la adhesión, proliferación y diferenciación de osteoblastos humanos (MG-63) in vitro [44]. Una capa de nanocomposite compuesto de BG y NPs de PCL, con diferentes contenidos de BG (1-90\% en peso) puede ser también aplicado sobre piezas de fosfato cálcico bifásico 
para mejorar las propiedades mecánicas (resistencia a la compresión en el rango de 0.2-1.45 MPa y módulos en el rango de 19.3-49.4 MPa) y la bioactividad; incremento de la degradación; e inducir a la diferenciación osteogénica de células humanas in vitro [47]. En otro trabajo, sustratos de titanio puro comercial y cables Kirschner (Kwires) de acero inoxidable fueron recubiertos con nanocomposites de poli(lactidaco-glicolato)-BG-HA preparados por medio de la fundición de solvente [66]. Estos estudios in vitro ofrecieron unas propiedades biológicas excelentes, como biomineralización, adhesión de células madre y viabilidad. Por otra parte, no fue detectado ningún signo de fallo en la adhesión entre el recubrimiento y el sustrato después de la implantación de los K-wires recubiertos dentro de la tibia de conejo, demostrando una fuerte unión entre el nanocomposite y los materiales metálicos.

Los nanocomposites de polímeros y BG están asumiendo un papel importante como membranas barrera para aplicaciones periodontales, con el objetivo de fomentar la regeneración periodontal por medio de un bloqueo físico de la migración de células epiteliales hacia el tejido dañado [67]. NPs de BG y alginato fueron propuestas porque, en comparación con las membranas de alginato, presentaban mejores propiedades in vitro: poca inflamación del tejido, degradación limitada, mejor biomineralización, buena adsorción de proteínas, y buena proliferación y adhesión celular de ligamentos fibroblásticos de periodontal humano (hPDLF) [60]. La introducción de NPs de BG en membranas de quitosano disminuían sus propiedades mecánicas, pero el mismo estudio demostraba la mejora de la bioactividad, actividad metabólica y mineralización por hPDLF y hBMSC [48].

Nanocomposites híbridos de polímero y BG, con los dos componentes indistinguibles por encima de la nanoescala, fueron recientemente fabricados por medio de procesos sol-gel: por medio de la disolución de polímeros preformados dentro de una solución precursora de sol-gel o bien formando simultáneamente tanto la fase orgánica como la inorgánica, a través de la polimerización sincronizada del monómero orgánico y del precursor sol-gel $[34,68]$. Dependiendo de las interacciones entre las dos fases, estos híbridos pueden ser clasificados dentro de los Tipo I, basados en conjuntos de redes, enlaces de hidrógeno o fuerzas de Van der Waals; y los híbridos de Tipo II, que poseen enlaces covalentes entre fases, además de otras interacciones y que, normalmente, son sintetizados por medio de la activación del polímero a través de un agente de acoplamiento al principio [68]. Biopolímeros como la gelatina, el poli $(\gamma-$ ácido glutámico) y el quitosano han sido investigados para fabricar polímeros para hacer híbridos del Tipo II con BG, mostrando un importante incremento en sus propiedades mecánicas cuando son comparados con las muestras monofásicas [49, 69, 70]. Utilizando gelatina e induciendo térmicamente a la separación de fases, pueden ser creados nanocomposites híbridos en estructuras nanofibrilares con contenido de sílice variable ( $0-30 \%$ peso), mostrando buenas propiedades biológicas in vitro en términos de biodegradabilidad estabilizada, formación de apatito y biocompatibilidad [43].

\section{LIBERACIÓN DE FÁRMACOS}

Además de las propiedades ya mencionadas del BG, se están haciendo esfuerzos científicos para explorar el potencial de liberación de fármacos que los sistemas basados en BG pueden tener. La técnica sol-gel es comúnmente empleada para producir sistemas de BG no mesoporoso y MBG para la liberación de medicamentos. Aplicaciones 
futuras podrían emplear los sistemas basados en BG como portadores de cápsulas y de la liberación controlada in situ de las moléculas bioactivas y los fármacos [71-73]. Estas moléculas pueden ser atrapadas en la red vítrea durante su fabricación bajo condiciones suaves, al igual que por medio de la técnica sol-gel, y entonces liberadas gradualmente en la disolución de BG. Una aproximación más versátil es inmovilizar las moléculas en la superficie del vidrio remojando el material de BG en una disolución de moléculas/medicamentos [71]. En esta segunda aproximación, químicos basados en el silanol o la fisisorción son utilizados comúnmente.

\section{Sistemas CONVEnCiOnaLes DE BG}

Diferentes clases de compuestos han sido estudiados para poder actuar como biomoléculas o fármacos integrables dentro de un sistema no mesoporoso de BG. El primer intento exitoso reportado fue de integración de antibióticos, la tetraciclina y la hidrocortisona, dentro de una solución sol-gel, y fue llevada a cabo a temperatura ambiente para no modificar las moléculas de los medicamentos [74, 75]. A pesar de que fue notable una liberación del $12 \%$ en las primeras 8 horas, tetraciclinas asociadas a $\beta$-ciclodextrinas seguían siendo liberadas después de 80 días in vitro, mientras que en los estudios in vivo mostraron una moderada reacción antinflamatoria [74]. En otro estudio, la tetraciclina era incorporada con hidrocortisona. Los resultados mostraron diferentes velocidades de liberación para los dos fármacos, pero no se encontraron interferencias de velocidades de liberación de un medicamento al otro [75].

Trabajos recientes estudian la integración de proteínas morfogénicas en el hueso (BMP-2 y BMP-9) así como otras proteínas usadas como modelos [57, 76, 77]. Microesferas de BG 45S5 fueron introducidas en un sistema de liberación de colágeno de base gel para BMPs [76]. Interesante, este sistema retiene más del 96\% de BMP-2 después de 1 hora, mientras que una matriz de esponja de colágeno común sólo retiene el 75\% después de 3 horas. Además, la versatilidad del sistema fue probada liberando otra molécula tipo BMP (BMP-9). El inhibidor de la tripsina, una molécula similar en tamaño a los BMPs, puede ser cargada dentro del BG en un proceso sol-gel de un solo paso, produciendo una liberación de dos etapas: una lenta inicial por unas horas y una segunda rápida durante semanas [77]. La BSA y la lisozima han sido introducidas satisfactoriamente como proteínas modelo en nanotubos de BG producidos por medio de electrospinning, conservando la bioactividad tanto del BG como de la lisozima [57].

\section{SISTEMAS MBG}

Las estructuras MBG están generando un especial interés para aplicaciones de liberación de fármacos, ya que su gran ordenación mesoporosa alberga una complicada red de cavidades que pueden albergar diferentes tipos de moléculas para su posterior liberación, demostrando su potencial como sistemas de liberación controlada [72, 73]. Para mejorar el control sobre los perfiles de carga y liberación es posible funcionalizar las paredes de los mesoporos con procesos basados en el silanol [78].

Los métodos de preparación son muy importantes para el control de las propiedades de los sistemas de liberación de medicamentos con base de MBG. Por ejemplo, probando diferentes compuestos como la metoclopramida o el triclosán como mo- 
delos de fármacos, el uso de surfactantes (P123, F127 o CTAB) conlleva a diferentes resultados en términos de volumen de poro y área específica, ambas propiedades influyen de una manera significativa la eficiencia de carga de los medicamentos [80,81]. La eficiencia de la carga y la velocidad de la liberación dependen de la composición de la pieza, como quedó demostrado con el perfil de liberación de la tetraciclina desde MBG con diferentes contenidos de $\mathrm{CaO}$ [79].

Considerando las muchas aplicaciones que podrían tener estos sistemas, pocos estudios se han centrado en las propiedades de estimulación de tejidos de las piezas 3D de MBG. En un modelo de ratón, el BMP-2 fue incorporado satisfactoriamente en una estructura macro/mesoporosa de BG dopada con $\mathrm{Ca} / \mathrm{Mg}$, mostrando mejores resultados que las piezas sin ninguna carga, refiriéndose a pruebas in vitro para la diferenciación osteogénica de rBMSCs e in vivo para la capacidad de formación de hueso ectópico [82]. Piezas de MBG que contienen boro han sido funcionalizadas con DEX, un conocido fármaco osteogénico, y analizadas in vitro, con resultados que muestran mayores actividades de ALP y mayores expresiones génicas de osteoblastos humanos relacionados con el proceso osteogénico (Col I, Runx2, ALP y BSP) [23]. En otro estudio, donde el VEGF era liberado desde estructuras de MBG [83], los resultados obtenidos mostraban una mejora en la viabilidad de las células endoteliales in vitro, lo que podría ser una buena estrategia para intentar evitar la limitada vascularización que se suele dar en el centro de los implantes ortopédicos.

La liberación sostenida de fármacos antibióticos desde estructuras de MBG ha sido extensamente investigada. Nanofibras de MBG producidas por medio de la técnica de electrospinning usando óxido de polietileno como agente separador de fases, mostraba unas prometedoras propiedades bioactivas y podría ser fácilmente utilizado como liberador de gentamicina [56]. Microesferas de MBG pueden ser fabricadas y empleadas para ser rellenadas y, posteriormente liberar triclosán con perfiles de velocidades modificables, dependiendo del tamaño de poro, a su vez relacionado con el método de preparación utilizado [81]. La ampicilina puede ser cargada en NPs de MBG mientras se conservan sus propiedades bactericidas, y así podría ser potencialmente utilizada como desinfectante para la limpieza de las raíces en el sistema de canales en tratamientos de endodoncias [84].

Las NPs de MBG también han sido utilizadas como sistemas de liberación de genes. NPs cargadas con pequeñas moléculas de ARN pueden ser fácilmente tomadas por las células, donde son capaces de regular el gen objetivo [85]. Estos resultados validan la utilización de NPs de MBG como un nuevo nanosistema de liberación de fármacos con un gran potencial de aplicación en ingeniería de tejidos óseos.

\section{Conclusiones}

Para mejorar la salud y la calidad de vida de pacientes con problemas ortopédicos o dentales, nuevas estrategias y materiales son necesarios para abordar las complejas cuestiones relacionadas con el medio dental y óseo. En este artículo, se han descrito brevemente materiales de BG nanoestructurados, resumiendo las propiedades químicas y los efectos potenciales de la disolución de productos en el comportamiento de las células. Sus aplicaciones potenciales como implantes de tejido duro y la liberación de fármacos fueron examinados con especial hincapié en la utilización del BG, tanto sólo como composite con otros componentes. 
El incremento en el número de artículos relacionados con este material evidencia el interés en el BG y está en continuo crecimiento, aunque las primeras composiciones de BG fueron creadas hace más de 40 años [1]. Además de las capacidades de fuerte adhesión al hueso y su bien caracterizada bioactividad debido a su disolución y su liberación de iones, las estructuras de BG se han visto nuevamente impulsadas gracias a la fabricación de MBG en 2006. Nuevas aplicaciones para el BG son ideadas para el tratamiento de problemas dentales y óseos, como traumas, infecciones o enfermedades como el cáncer o la osteoporosis [3, 72, 73]. Combinando las oportunidades de los experimentos a diferentes escalas (nano, meso, micro y macro) puede ser la clave para el diseño de nuevos biomateriales capaces de interactuar con complejos celulares en el interior del organismo y tener así una completa y rápida recuperación del paciente.

\section{REFERENCIAS BIBLIOGRÁFICAS}

[1] Hench, L.L. "The story of bioglass". J Mater Sci Mater Med, 2006. 17(11): 967-78.

[2] Jones, J.R. "Review of bioactive glass: From hench to hybrids". Acta Biomater, 2012.

[3] Yan, X. et al. "Highly ordered mesoporous bioactive glasses with superior in vitro bone-forming bioactivities". Angew Chem Int Ed Engl, 2004. 43(44): 5980-4.

[4] Tran, N. y T.J. Webster. "Nanotechnology for bone materials". Wiley Interdisciplinary Reviews: Nanomedicine and Nanobiotechnology, 2009. 1(3): 336-351.

[5] Tomsia, A.P. et al. "Nanotechnology approaches for better dental implants". The International Journal of Oral \& Maxillofacial Implants, 2011. 26(Suppl): 25-49.

[6] Tomsia, A.P. et al. "Nanotechnology for dental implants". Oral \& Craniofacial Tissue Engineering, 2012. 2(1): 23-34.

[7] Dvir, T. et al. "Nanotechnological strategies for engineering complex tissues". Nat Nanotechnol, 2011. 6(1): 13-22.

[8] Moghimi, S.M., A.C. Hunter, y J.C. Murray. "Nanomedicine: Current status and future prospects". FASEB J, 2005. 19(3): 311-30.

[9] Li, R., A.E. Clark y L.L. Hench. "An investigation of bioactive glass powders by sol-gel processing". J Appl Biomater, 1991. 2(4): 231-9.

[10] Sepulveda, P., J.R. Jones y L.L. Hench. "Characterization of melt-derived 45 S5 and solgel-derived 58 S bioactive glasses”. J Biomed Mater Res, 2001. 58(6): 734-40.

[11] Fu, Q. et al. "Bioactive glass scaffolds for bone tissue engineering: state of the art and future perspectives". Mater Sci Eng C Mater Biol Appl, 2011. 31(7): 1245-1256.

[12] Rahaman, M.N. et al. "Bioactive glass in tissue engineering”. Acta Biomater, 2011. 7(6): 2355-73.

[13] Wu, C. et al. "A comparative study of mesoporous glass/silk and non-mesoporous glass/silk scaffolds: physiochemistry and in vivo osteogenesis". Acta Biomater, 2011. 7(5): 2229-36.

[14] Wu, C. et al. "The effect of mesoporous bioactive glass on the physiochemical, biological and drug-release properties of poly(DL-lactide-co-glycolide) films". Biomaterials, 2009. 30(12): 2199-208.

[15] Cormack, A.N., The structure of bioactive glasses and their surfaces, in bio-glasses. 2012, John Wiley \& Sons, Ltd. 65-74. 
[16] Mercier, C. et al. "Influence of P2O5 content on the structure of SiO2-Na2O-CaOP205 bioglasses by 29Si and 31P MAS-NMR”. Journal of Non-Crystalline Solids, 2011. 357(24): 3901-3909.

[17] Hill, R.G. y D.S. Brauer. "Predicting the bioactivity of glasses using the network connectivity or split network models". Journal of Non-Crystalline Solids, 2011. 357(24): 3884-3887.

[18] Hench, L.L. y J.M. Polak. "Third-generation biomedical materials". Science, 2002. 295(5557): 1014-7.

[19] Hench, L.L. "Bioceramics". Journal of the American Ceramic Society, 1998. 81(7): 17051728.

[20] Hench, L.L., I.D. Xynos y J.M. Polak. "Bioactive glasses for in situ tissue regeneration". Journal of Biomaterials Science, Polymer Edition, 2004. 15(4): 543-562.

[21] Hoppe, A., N.S. Guldal y A.R. Boccaccini. "A review of the biological response to ionic dissolution products from bioactive glasses and glass-ceramics". Biomaterials, 2011. 32(11): 2757-74.

[22] Wang, X. et al. "Synthesis and characterization of hierarchically macroporous and mesoporous CaO-MO-SiO(2)-P(2)O(5) (M=Mg, Zn, Sr) bioactive glass scaffolds". Acta Biomater, 2011. 7(10): 3638-44.

[23] Wu, C. et al. "Proliferation, differentiation and gene expression of osteoblasts in boron-containing associated with dexamethasone deliver from mesoporous bioactive glass scaffolds". Biomaterials, 2011. 32(29): 7068-78.

[24] Wu, C. et al. "Multifunctional magnetic mesoporous bioactive glass scaffolds with a hierarchical pore structure". Acta Biomater, 2011. 7(10): 3563-72.

[25] Zhu, Y. et al. "The effect of zirconium incorporation on the physiochemical and biological properties of mesoporous bioactive glasses scaffolds". Microporous and Mesoporous Materials, 2011. 143(2-3): 311-319.

[26] Wu, C. et al. "Hypoxia-mimicking mesoporous bioactive glass scaffolds with controllable cobalt ion release for bone tissue engineering". Biomaterials, 2012. 33(7): 207685.

[27] Wu, C. et al. "Copper-containing mesoporous bioactive glass scaffolds with multifunctional properties of angiogenesis capacity, osteostimulation and antibacterial activity". Biomaterials, 2013. 34(2): 422-33.

[28] Han, P. et al. "The cementogenic differentiation of periodontal ligament cells via the activation of Wnt/beta-catenin signalling pathway by Li+ ions released from bioactive scaffolds". Biomaterials, 2012. 33(27): 6370-9.

[29] Hupa, L. y A. Yli-Urpo. Dental applications of glasses, in bio-glasses. 2012, John Wiley \& Sons, Ltd. 159-175.

[30] Bellantone, M., H.D. Williams, y L.L. Hench. "Broad-spectrum bactericidal activity of Ag20-doped bioactive glass". Antimicrobial Agents and Chemotherapy, 2002. 46(6): 1940-1945.

[31] El-Kady, A.M. et al. "Synthesis, characterization and microbiological response of silver doped bioactive glass nanoparticles". Ceramics International, 2012. 38(1): 177-188.

[32] Navarro, M. et al. "Biomaterials in orthopaedics". J R Soc Interface, 2008. 5(27): 113758.

[33] Boccaccini, A.R. et al. "Polymer/bioactive glass nanocomposites for biomedical applications: A review”. Composites Science and Technology, 2010. 70(13): 1764-1776. 
[34] Valliant, E.M. y J.R. Jones. "Softening bioactive glass for bone regeneration: sol-gel hybrid materials". Soft Matter, 2011. 7(11): 5083.

[35] Langer, R. y J. Vacanti. “Tissue engineering”. Science, 1993. 260(5110): 920-926.

[36] Fu, Q., E. Saiz, y A.P. “Tomsia, bioinspired strong and highly porous glass scaffolds”. Adv Funct Mater, 2011. 21(6): 1058-1063.

[37] Fu, Q., E. Saiz, y A.P. Tomsia. "Direct ink writing of highly porous and strong glass scaffolds for load-bearing bone defects repair and regeneration". Acta Biomater, 2011. $7(10): 3547-54$.

[38] Garcia, A. et al. "Preparation of 3-D scaffolds in the SiO2-P2O5 system with tailored hierarchical meso-macroporosity". Acta Biomater, 2011. 7(3): 1265-73.

[39] Wu, C. et al. "Three-dimensional printing of hierarchical and tough mesoporous bioactive glass scaffolds with a controllable pore architecture, excellent mechanical strength and mineralization ability". Acta Biomater, 2011. 7(6): 2644-50.

[40] Liu, X. et al. "Porous and strong bioactive glass (13-93) scaffolds prepared by unidirectional freezing of camphene-based suspensions". Acta Biomater, 2012. 8(1): 415-23.

[41] Wu, Z.Y. et al. "Melt-derived bioactive glass scaffolds produced by a gel-cast foaming technique". Acta Biomater, 2011. 7(4): 1807-16.

[42] Midha, S. et al. "Bioactive glass foam scaffolds are remodelled by osteoclasts and support the formation of mineralized matrix and vascular networks in vitro". Advanced Healthcare Materials, 2012: n/a-n/a.

[43] Drnovsek, N. et al. "Bioactive glass enhances bone ingrowth into the porous titanium coating on orthopaedic implants". Int Orthop, 2012. 36(8): 1739-45.

[44] Liu, W., S. Thomopoulos y Y. Xia. "Electrospun nanofibers for regenerative medicine". Adv Healthc Mater, 2012. 1(1): 10-25.

[45] Polini, A. et al. "Osteoinduction of human mesenchymal stem cells by bioactive composite scaffolds without supplemental osteogenic growth factors". PLoS One, 2011. 6(10): e26211.

[46] Lu, H. et al. "Electrospun submicron bioactive glass fibers for bone tissue scaffold". J Mater Sci Mater Med, 2009. 20(3): 793-8.

[47] Hong, Y. et al. "Fabrication and drug delivery of ultrathin mesoporous bioactive glass hollow fibers". Advanced Functional Materials, 2010. 20(9): 1503-1510.

[48] Xie, J., E.R. Blough y C.H. Wang. "Submicron bioactive glass tubes for bone tissue engineering". Acta Biomater, 2012. 8(2): 811-9.

[49] Quintero, F. et al. "Laser spinning of bioactive glass nanofibers". Advanced Functional Materials, 2009. 19(19): 3084-3090.

[50] Maèkoviæ, M. et al. "Bioactive glass (type 45S5) nanoparticles: in vitro reactivity on nanoscale and biocompatibility". Journal of Nanoparticle Research, 2012.14(7).

[51] Lei, B. et al. "Versatile fabrication of nanoscale sol-gel bioactive glass particles for efficient bone tissue regeneration". Journal of Materials Chemistry, 2012. 22(33): 16906.

[52] Srinivasan, S. et al. "Biocompatible alginate/nano bioactive glass ceramic composite scaffolds for periodontal tissue regeneration". Carbohydrate Polymers, 2012. 87(1): 274-283.

[53] Luz, G.M. y J.F. Mano. "A nanotectonics approach to produce hierarchically organized bioactive glass nanoparticles-based macrospheres". Nanoscale, 2012. 4(20): 6293-7.

[54] Wang, Z. et al. "The dentine remineralization activity of a desensitizing bioactive glass-containing toothpaste: An in vitro study". Aust Dent J, 2011. 56(4): 372-81. 
[55] Chiang, Y.C. et al. "A novel mesoporous biomaterial for treating dentin hypersensitivity". J Dent Res, 2010. 89(3): 236-40.

[56] $\mathrm{Xu}, \mathrm{C}$. et al. "Biocompatibility and osteogenesis of biomimetic bioglass-collagenphosphatidylserine composite scaffolds for bone tissue engineering". Biomaterials, 2011. 32(4): 1051-8.

[57] Marelli, B. et al. "Accelerated mineralization of dense collagen-nano bioactive glass hybrid gels increases scaffold stiffness and regulates osteoblastic function". Biomaterials, 2011. 32(34): 8915-26.

[58] Kim, H.W., J.H. Song y H.E. Kim. "Bioactive glass nanofiber-collagen nanocomposite as a novel bone regeneration matrix". J Biomed Mater Res A, 2006. 79(3): 698-705.

[59] Lin, H.M., Y.H. Lin y F.Y. Hsu. "Preparation and characterization of mesoporous bioactive glass/polycaprolactone nanofibrous matrix for bone tissues engineering". J Mater Sci Mater Med, 2012.

[60] Gerhardt, L.C. et al. "The pro-angiogenic properties of multi-functional bioactive glass composite scaffolds". Biomaterials, 2011. 32(17): 4096-108.

[61] Misra, S.K. et al. "Effect of nanoparticulate bioactive glass particles on bioactivity and cytocompatibility of poly(3-hydroxybutyrate) composites". J R Soc Interface, 2010. 7(44): 453-65.

[62] Roohani-Esfahani, S.I. et al. "Effects of bioactive glass nanoparticles on the mechanical and biological behavior of composite coated scaffolds". Acta Biomater, 2011. 7(3): 1307-18.

[63] Mehdikhani-Nahrkhalaji, M. et al. "Novel nanocomposite coating for dental implant applications in vitro and in vivo evaluation". J Mater Sci Mater Med, 2012. 23(2): 48595.

[64] Ivanovski, S. “Periodontal regeneration”. Aust Dent J, 2009. 54 Suppl 1: S118-28.

[65] Mota, J. et al. "Chitosan/bioactive glass nanoparticle composite membranes for periodontal regeneration". Acta Biomater, 2012. 8(11): 4173-80.

[66] Novak, B.M. "Hybrid Nanocomposite Materials? Between inorganic glasses and organic polymers". Advanced Materials, 1993. 5(6): 422-433.

[67] Mahony, 0. et al. "Silica-gelatin hybrids with tailorable degradation and mechanical properties for tissue regeneration". Advanced Functional Materials, 2010. 20(22): 3835-3845.

[68] Poologasundarampillai, G. et al. "Bioactive silica-poly( $\gamma$-glutamic acid) hybrids for bone regeneration: effect of covalent coupling on dissolution and mechanical properties and fabrication of porous scaffolds". Soft Matter, 2012. 8(17): 4822.

[69] Yang, B. et al. "Preparation and characterization of a novel chitosan scaffold". Carbohydrate Polymers, 2010. 80(3): 860-865.

[70] Lei, B. et al. "Nanofibrous gelatin-silica hybrid scaffolds mimicking the native extracellular matrix (ECM) using thermally induced phase separation". Journal of Materials Chemistry, 2012. 22(28): 14133.

[71] Hum, J. y A.R. "Boccaccini, bioactive glasses as carriers for bioactive molecules and therapeutic drugs: a review". J Mater Sci Mater Med, 2012. 23(10): 2317-33.

[72] Vallet-Regi, M., I. Izquierdo-Barba y M. Colilla. "Structure and functionalization of mesoporous bioceramics for bone tissue regeneration and local drug delivery". Philos Transact A Math Phys Eng Sci, 2012. 370(1963): 1400-21. 
[73] Wu, C. y J. Chang. "Mesoporous bioactive glasses: Structure characteristics, drug/ growth factor delivery and bone regeneration application". Interface Focus, 2012. 2(3): 292-306.

[74] Domingues, Z.R. et al. "Bioactive glass as a drug delivery system of tetracycline and tetracycline associated with $\beta$-cyclodextrin". Biomaterials, 2004. 25(2): 327-333.

[75] Andrade, A.L. et al. "Tetracycline and/or hydrocortisone incorporation and release by bioactive glasses compounds". Journal of Non-Crystalline Solids, 2009. 355(13): 811816.

[76] Bergeron, E. et al. "Differentiation of preosteoblasts using a delivery system with BMPs and bioactive glass microspheres". J Mater Sci Mater Med, 2007. 18(2): 255-63.

[77] Santos, E.M., S. Radin y D.P. “Sol-gel derived carrier for the controlled release of proteins". Biomaterials, 1999. 20(18): 1695-1700.

[78] Hoffmann, F. et al. "Silica-based mesoporous organic-inorganic hybrid materials". Angew Chem Int Ed Engl, 2006. 45(20): 3216-51.

[79] Zhao, Y.F. et al. "In situ SAXRD study of sol-gel induced well-ordered mesoporous bioglasses for drug delivery". J Biomed Mater Res A, 2008. 85(4): 1032-42.

[80] Arcos, D. et al. "Ordered mesoporous microspheres for bone grafting and drug delivery". Chemistry of Materials, 2009. 21(6): 1000-1009.

[81] Zhao, L. et al. "Mesoporous bioactive glasses for controlled drug release". Microporous and Mesoporous Materials, 2008. 109(1-3): 210-215.

[82] Dai, C. et al. "Osteogenic evaluation of calcium/magnesium-doped mesoporous silica scaffold with incorporation of rhBMP-2 by synchrotron radiation-based muCT". Biomaterials, 2011. 32(33): 8506-17.

[83] Wu, C. et al. "Mesoporous bioactive glass scaffolds for efficient delivery of vascular endothelial growth factor". J Biomater Appl, 2012.

[84] Fan, W. et al. "Porous Ca-Si-based nanospheres: A potential intra-canal disinfectantcarrier for infected canal treatment". Materials Letters, 2012. 81: 16-19.

[85] El-Fiqi, A. et al. "Capacity of mesoporous bioactive glass nanoparticles to deliver therapeutic molecules". Nanoscale, 2012. 